\title{
Optimal Forest Stand Aggregation and Harvest Scheduling Using Compactly Formulated Integer Programming
}

\author{
Atsushi Yoshimoto ${ }^{1 *}$, Patrick Asante ${ }^{2}$, Masashi Konoshima ${ }^{3}$
}

Abstract:

We propose an alternative approach for optimal forest stand aggregation for implementing harvest scheduling, which allows for multiple harvests using a compact formulated integer programming that seeks an optimal aggregated pattern among candidates for forest management units over the planning horizon. We deal with aggregation of small forest stands by introducing the concept of a "hyper unit" as a possible aggregated management unit, which is predefined with the use of adjacency relationship among the set of forest stands. Our proposed approach is based on an optimization framework of a traditional spatially constrained harvest scheduling problem which is used to choose the best set of treatments for the aggregated management units, as well as the original un-aggregated forest stands, while allowing for multiple harvests. We also apply adjacency constraints to create aggregated management units, which are separated from other units, as well as un-aggregated forest stands such that, no adjacent units are harvested in the same period.

Keywords: forest management, harvest scheduling, adjacency, unit aggregation, integer programming

\section{Introduction}

As a result of small-scale and spatially fragmented distribution of forest stands for harvesting and thinning operations, as well as the prolonged timber price slump, improving management efficiency by reducing management cost has been one of the main concerns for Japanese forest policy makers. In 2012, the Japanese government introduced a newly enforceable forest management planning system (MAFF, 2012). This new management planning system aims to improve the efficiency of forest practices, as well as facilitate multiple functions and sustainable management through the management of aggregated forest areas.

Aggregating adjacent small forest stands into a larger management unit (so-called "Danchi-ka" in Japanese) has received a great deal of attention as one of the efforts to achieve low cost forest management with governmental subsidy incentive. It allows forest owners to build a large-scale network of forest logging roads for delivery of logs as well as periodic maintenance and a costeffective thinning process. This could ultimately lead to a reduction in timber production cost as a whole. Aggregation can also provide small-scale forest landowners, the opportunity to partner with other member landowners involved in forest aggregation, so as to share the cost of running vehicles and other heavy-equipments. In addition, aggregation of forest stands also plays an important role in maintaining and protecting corridors that are critical habitats for certain wildlife species.

The conventional way of addressing these types of problems, which was first introduced in the 1980's and early the 90's, cannot be used to guarantee the connectivity or clustering of forest stands. Because of this, an additional consideration was introduced to include the aggregation of adjacent forest stands into a larger management unit. For environmental and legislative reasons, it may be necessary to limit the size of these management units. This can be done by using a green-up constraint. The aim of this constraint is to limit the size of the contiguous areas that have been recently cut, by "freezing" the cutting of cutblocks adjacent to the one just cut until a certain greenup period of time has passed. In other words, once this maximum harvest block size is reached, none of the surrounding forest may be harvested until regrowth in the clearcut area has reached the green-up period. For forest dependent wildlife species, green-up constraints may provide hiding cover for security from predators or thermal cover for protection against the effects of weather (British Columbia Ministry of Forests, 1999). Such regulations may be easy to legislate, but very difficult to incorporate into an optimization model.

Several authors have incorporated green-up constraints in an optimization model, to enforce green recovery or continuity of crown coverage of forest stands under harvesting as an indirect

Received Nov. 1, 2016; Accepted Mar. 3, 2017

1 The Institute of Statistical Mathematics, Japan

${ }^{2}$ Ministry of Forests, Lands and Natural Resources Operations Government of British Columbia, Canada

${ }^{3}$ University of the Ryukyus, Japan

*Corresponding Author: yoshimoa@ism.ac.jp 
aggregation (Yoshimoto, 2001; Boston and Bettinger, 2002; 2006; Yoshimoto and Konoshima, 2015). A review of literature on this subject shows that, Goycoolea et al. (2009) coined the terms, dynamic and static green-up to describe green-up constraints. Dynamic green-up constraints are applied to forest stands while static green-up are applied to clusters or aggregated units. In this paper, we make no distinction between the two types since it is unnecessary for formulation purposes.

Common aggregation requires integration of direct adjacent forest stands and indirect adjacent forest stands, to create a set of aggregated forest management units. Murray (1999) referred this problem as an area restriction model (ARM) as opposed to conventional adjacency problems. Several studies have addressed ARM-type problems with an exact method, including McDill et al. (2002), Murray and Weintraub (2002), Rebain and McDill (2003), Goycoolea et al. (2005). These efforts, however, have limited ability to address harvest scheduling with multiple harvests over the planning horizon.

In this paper, with the use of the common matrix algebra as in Yoshimoto and Konoshima (2015), we propose an alternative approach for optimal forest stand aggregation using compactly formulated programming allowing for multiple harvests over time. The concept of Model I (Johnson and Scheurman 1977) is applied to deal with multiple harvests over time in the integer programming formulation as well as the concept of the general management unit approach (McDill et al., 2002; Goycoolea et al., 2005) to enumerate feasible aggregated units (called "hyper units" hereafter) for forest stand aggregation. The matrix notation is used as the general expression of compactness to define decision variables in Model I as well as adjacency among forest units and "hyper units". Difference between the present work and others is that our problem is formulated compactly, which is based on matrix notation, so that extension of the formulation becomes rather easy. By modifying the elements of the defined matrices, non-forestry systems analysts can produce the harvest scheduling system for all practical purposes.

In the section that follows, we introduce a new formulation of optimal forest stand aggregation for implementing harvest scheduling which allows for multiple harvests using a compact formulated integer programming. This is then followed by a demonstrative presentation in section three. We end with brief concluding remarks in the final section.

\section{Problem Specification for Forest Stand Aggregation}

We assume the objective is to maximize the net present value of total profit from harvesting activities over the planning horizon. Constraints include land accounting, harvest flow, and spatial restrictions to avoid harvesting two adjacent stands during the same period. Unlike the methods introduced by McDill et al. (2002), Murray and Weintraub (2002), Goycoolea et al. (2005), and Rebain and McDill (2003), we build on the Model I formulation and concept of adjacency. Our approach consists of four steps: 1) create candidate "hyper units"-predefined before solution search starts-for forest stand aggregation based on each forest stand (distinguish this as "base unit" hereafter) and define the decision variable matrix considering the base and hyper units, 2) develop adjacency constraints for the set of base and hyper units, 3) develop the extended land accounting constraints by introducing an overlapping relationship among the base and hyper units, and 4) formulate a forest stand aggregation harvest scheduling problem to allow for multiple harvests.

Our four-step process begins with creation of candidate hyper units for aggregation. In this paper, we create one hyper unit from one base unit following a pre-specified rule. The applied rule is to develop a hyper unit from one base unit (called the "target unit" since it is the only candidate for this process) by connecting other base units within a certain distance. This rule can be implemented by creating a buffer zone from the center of the target unit. If a base unit is within or at least part of the buffer zone from the target unit, then such a unit is aggregated into one hyper unit created for the target unit.

Let $\boldsymbol{X}=\left(\boldsymbol{x}_{1}, \ldots, \boldsymbol{x}_{m}\right)^{\prime}=\left(\tilde{\boldsymbol{x}}_{1}, \ldots, \tilde{\boldsymbol{x}}_{n}\right)$ be an $(m \times n)$ binary decision variable matrix with $m$ as the number of base units and $n$ as the number of treatments defined for forest stands, and ' denotes the transpose where $\boldsymbol{x}_{i}$ is the $i$-th row vector of $\boldsymbol{X}$ for the $i$-th base unit and $\tilde{\boldsymbol{x}}_{j}$ is the $j$-th column vector for harvesting at the $j$-th treatment. An element of $\boldsymbol{X}$ is thus defined by, $x_{i j}$, which becomes 1 if the $j$-th treatment is implemented for the $i$-th base unit and 0 if not. The corresponding coefficient, $c_{i j}$, represents the net present value of the profit from implementing the $j$-th treatment to the $i$-th base unit. We now introduce two types of index sets - the base unit set, $\mathrm{u}_{i}=\{i\}$, including only the index 
for its own unit, and the hyper unit set, $\mathrm{HU}_{i}=$ indecies combined for a hyper unit based on $\mathrm{u}_{i}$.

With the decision variable matrix defined for the base units by $\boldsymbol{X}$, the $(m \times n)$ binary decision variable matrix, $\boldsymbol{Y}$, for the set of hyper units consists of its element, $y_{i j}$, which becomes 1 if the $j$-th treatment is implemented for $\mathrm{HU}_{i}$ and 0 if not. Combining these two binary decision variable matrices, $(\boldsymbol{X}, \boldsymbol{Y})$, we introduce a new binary decision variable matrix for the base and hyper units together, $\boldsymbol{W}=(\boldsymbol{X}, \boldsymbol{Y})^{\prime}$ with a $(2 m \times n)$ dimension. This is the binary decision variable matrix used for our formulation of forest stand aggregation.

We now introduce adjacency constraints for the base and hyper units with the above decision matrix, $\boldsymbol{W}$. As mentioned in Yoshimoto and Konoshima (2015), two adjacency matrices, a spatial adjacency matrix, and an activity adjacency matrix are needed for allowing multiple harvests within the concept of treatments. The spatial adjacency matrix is created by observing the adjacency relationship between the base and hyper units for the index sets in $\left\{\mathrm{HU}_{i}\right\}$ and $\left\{\mathrm{NB}_{i}\right\}$, where $\mathrm{NB}_{i}$ is the index set of adjacent base units to the $i$-th base unit. The resultant spatial adjacency matrix consists of four locally spatial adjacency matrices:

$$
\boldsymbol{A}^{S}=\left(\begin{array}{cc}
\boldsymbol{A}_{\mathrm{u}-\mathrm{u}} & \boldsymbol{A}_{\mathrm{u}-\mathrm{HU}} \\
\boldsymbol{A}_{\mathrm{HU}-\mathrm{u}} & \boldsymbol{A}_{\mathrm{HU}-\mathrm{HU}}
\end{array}\right) \quad(2 m \times 2 m)
$$

where $\boldsymbol{A}_{\mathrm{u}-\mathrm{u}}$ is the spatial adjacency matrix among the base units, $\boldsymbol{A}_{\mathrm{u}-\mathrm{HU}}\left(=\boldsymbol{A}_{\mathrm{HU}-\mathrm{u}}^{\prime}\right), \boldsymbol{A}_{\mathrm{HU}-\mathrm{u}}$ is the spatial adjacency matrix among the base and hyper units, and $\boldsymbol{A}_{\mathrm{HU}-\mathrm{HU}}$ is the spatial adjacency matrix among hyper units. Note that overlapping is not considered as adjacency unlike Goycoolea et al. (2005). An element of $\boldsymbol{A}_{\mathrm{u}-\mathrm{u}}$ is defined by:

$$
a_{i j}^{\mathrm{s}}=\left\{\begin{array}{ll}
1 & \text { if } j \in \mathrm{NB} \\
0 & \text { if } j \notin \mathrm{NB}_{i}
\end{array}, \quad \forall_{i \neq j}\right.
$$

with $a_{i i}^{\mathrm{s}}=0\left(\right.$ for $\left.{ }^{\forall} i\right)$ and an element of $\boldsymbol{A}_{\mathrm{u}-\mathrm{HU}}$ is defined by:

$$
a_{i j}^{\mathrm{u}-\mathrm{HU}}= \begin{cases}1 & \text { if } \mathrm{u}_{i} \in \underset{k \in \mathrm{HU}_{j}}{\bigcup} \mathrm{NB}_{k} \& \mathrm{u}_{i} \cap \mathrm{HU}_{j}=\varnothing \\ 0 & \text { otherwise }\end{cases}
$$

while an element of $\boldsymbol{A}_{\mathrm{HU}-\mathrm{HU}}$ is:

$$
a_{i j}^{\mathrm{HU}-\mathrm{HU}}= \begin{cases}1 & \text { if } \mathrm{HU}_{i} \cap \bigcup_{k \in \mathrm{HU}_{j}} \mathrm{NB}_{k} \neq \varnothing \& \mathrm{HU}_{i} \cap \mathrm{HU}_{j}=\varnothing \\ 0 & \text { otherwise }\end{cases}
$$

An element, $a_{i j}^{v}$, of the activity adjacency matrix, $\boldsymbol{A}^{V}$, reflects the concurrent relationship between the $i$-th and $j$-th treatments. If harvest occurs during the same period for both treatments, the value of $a_{i j}^{v}$ becomes 1. It is also possible to incorporate the green-up constraints which restrict both the size of the openings and the length of time before adjacent forest management units are harvested. Green-up can be handled by the activity adjacency matrix introduced by Yoshimoto and Konoshima (2015). It can be applied to hyper units as well as base units at the same time. Goycoolea et al. (2009) distinguished dynamic and static green-up constraints when applying them to either adjacent forest stands or aggregated units. However, given the above concept of formulation for adjacency, this distinction becomes very unnecessary.

$$
a_{i j}^{v}= \begin{cases}1 & \text { if the } i \text {-th and } j \text {-th treatments have any concurrent harvest } \\ 0 & \text { otherwise }\end{cases}
$$

Note that the diagonal elements become 1, i.e. $a_{i i}^{\mathrm{v}}=1$ (for ${ }^{\forall} i$ ).

Combining the spatial and activity adjacency matrices with the decision variable matrix, $\boldsymbol{W}$, we obtain the following adjacency constraints (see Yoshimoto and Konoshima (2015) for the detail explanation): 


$$
\left[\tilde{\boldsymbol{A}}+\operatorname{diag}\left(\tilde{\boldsymbol{A}} \cdot \mathbf{1}_{2 m n}\right)\right] \cdot \operatorname{vec}\left(\boldsymbol{W}^{\prime}\right) \leq \tilde{\boldsymbol{A}} \cdot \mathbf{1}_{2 m n}
$$

where $\mathbf{1}_{2 m n}$ is the $2 m n$ dimensional unit vector, $\operatorname{diag}\left(\tilde{\boldsymbol{A}} \cdot \mathbf{1}_{2 m n}\right)$ is to place a vector, $\tilde{\boldsymbol{A}} \cdot \mathbf{1}_{2 m n}=$ $\left(\tilde{a}_{1}, \tilde{a}_{2}, \ldots, \tilde{a}_{2 m n}\right)^{\prime}$, into a diagonal matrix whose diagonal entries starting in the upper left corner are $\left(\tilde{a}_{1}, \tilde{a}_{2}, \ldots, \tilde{a}_{2 m n}\right)$, vec(.) is vectorization operand, and $(2 m n \times 2 m n)$ matrix, $\tilde{\boldsymbol{A}}=\boldsymbol{A}^{S} \otimes \boldsymbol{A}^{V}$ with Kronecker product by $\otimes$. Note that not all base units are aggregated into hyper units because some base units must remain for harvest by itself. Although forest managers can choose to harvest these base units along with hyper units, these base units cannot be harvested if their adjacent hyper units or base units are harvested. The tighter adjacency constraints can be obtained by Kronecker product of spatial adjacency matrix and $n$ dimensional unit vector:

$$
\left[\tilde{\boldsymbol{A}}+\operatorname{diag}\left(\tilde{\boldsymbol{A}} \cdot \mathbf{1}_{2 m n}\right)\right] \cdot \operatorname{vec}\left(\boldsymbol{W}^{\prime}\right) \leq\left(\boldsymbol{A}^{S} \cdot \mathbf{1}_{2 m}\right) \otimes \mathbf{1}_{n}
$$

This is because at most one treatment can be selected for one base unit handled by the extended land accounting constraints described below.

The land accounting constraints avoid overlapping treatments for a given base unit. Thus, overlapping between base and hyper units can be addressed in the same way. Let us now introduce two matrices to describe 1) the overlapping relationship of treatments among the base units by an $(m \times m)$ matrix, $\boldsymbol{O}^{\mathrm{u}}$, and 2) overlapping among the base units and hyper units by an $(m \times m)$ matrix, $\boldsymbol{O}^{\mathrm{H}}$. An element of $\boldsymbol{O}^{\mathrm{u}}$ is defined between $\mathrm{u}_{i}$ and $\mathrm{u}_{j}$ by:

$$
o_{i j}^{\mathrm{u}}=\left\{\begin{array}{l}
1 \text { if } \mathrm{u}_{i} \cap \mathrm{u}_{j} \neq \varnothing \\
0 \text { if } \mathrm{u}_{i} \cap \mathrm{u}_{j}=\varnothing
\end{array}\right.
$$

and between $\mathrm{u}_{i}$ and $\mathrm{HU}_{j}$, an element of $\boldsymbol{O}^{\mathrm{H}}$ is as follows:

$$
o_{i j}^{\mathrm{H}}=\left\{\begin{array}{l}
1 \text { if } \mathrm{u}_{i} \cap \mathrm{HU}_{j} \neq \varnothing \\
0 \text { if } \mathrm{u}_{i} \cap \mathrm{HU}_{j}=\varnothing
\end{array}\right.
$$

Combining the above two matrices, the overlapping matrix for the base and hyper units is obtained for the decision variable matrix, $\boldsymbol{W}$ by a $(m \times 2 m)$ matrix of $\boldsymbol{O}=\left(\boldsymbol{O}^{\mathrm{u}}, \boldsymbol{O}^{\mathrm{H}}\right)$. Thus the extended land accounting constraints become:

$$
\left(\boldsymbol{O} \otimes \mathbf{1}_{n}^{\prime}\right) \cdot \operatorname{vec}\left(\boldsymbol{W}^{\prime}\right) \leq \mathbf{1}_{m}
$$

This only allows at most one treatment for one base unit, which excludes overlapping of hyper units for base units as well.

The volume flow constraints control fluctuation of volume flow level over the planning periods. Let us first introduce an $(m \times n)$ volume flow matrix at period $p$ by $\boldsymbol{V}_{p},(p=1, \ldots, T)$, whose element, $v_{i, j}^{p}$, is the volume flow at period $p$ by assigning the $j$-th treatment to the $i$-th forest stand, $x_{i, j}=1$. The resultant obtainable volume at period from can be expressed by:

$$
\operatorname{tr}\left(\boldsymbol{V}_{p}{ }^{\prime} \boldsymbol{X}\right)=\sum_{i=1}^{m} \sum_{j=1}^{n} v_{i, j}^{p} \cdot x_{i, j}, \quad p=1, \ldots, T
$$

The volume flow matrix for $\boldsymbol{W}$ at period $p$ needs to be arranged by $\tilde{\boldsymbol{V}}_{p}=\left(\boldsymbol{V}_{p}, \overline{\boldsymbol{V}}_{p}\right)^{\prime}$ with $(2 m \times n)$, where $\overline{\boldsymbol{V}}_{p}$ is the volume flow matrix for $\boldsymbol{Y}$ at period $p$. An element of $\overline{\boldsymbol{V}}_{p}$ is calculated by:

$$
\bar{v}_{i j}^{p}=\sum_{k \in \mathrm{HU}_{i}} v_{k j}^{p}
$$

Thus the volume flow constraints become: 


$$
(1-\alpha) v_{0} \leq \operatorname{tr}\left(\tilde{\boldsymbol{V}}_{p}^{\prime} \cdot \boldsymbol{W}\right) \leq(1+\alpha) v_{0}, \quad p=1, \ldots, T
$$

where $v_{0}$ is a given flow level and $\alpha$ is fluctuation, which ensures that volume flow at every period stays within $\pm \alpha$ from $v_{0}$. Note that $\operatorname{tr}(\boldsymbol{A})$ is a trace of a square matrix $\boldsymbol{A}$, which is the sum of the elements on the main diagonal (the diagonal from the upper left to the lower right) of the given matrix $\boldsymbol{A}$.

To specify the objective function of the forest stand aggregation problem, a coefficient matrix for $\boldsymbol{W}$ becomes $\tilde{\boldsymbol{C}}=(\boldsymbol{C}, \overline{\boldsymbol{C}})^{\prime}$ with $(2 m \times n)$, where $\boldsymbol{C}$ is a coefficient matrix for the decision variable matrix, $\boldsymbol{X}$, and $\overline{\boldsymbol{C}}$ is that for $\boldsymbol{Y}$. If any reward is used for aggregation to create a hyper unit, a coefficient, $\bar{c}_{i j}$ for $y_{i j}$ can be defined by:

$$
\bar{c}_{i j}=(1+r) \sum_{k \in \mathrm{HU}_{i}} c_{k j}
$$

where $r$ is a reward rate. Thus, the objective function is expressed by:

$$
Z=\max _{W} \operatorname{tr}\left(\tilde{\boldsymbol{C}}^{\prime} \cdot \boldsymbol{W}\right)=\sum_{i=1}^{m} \sum_{j=1}^{n}\left(c_{i j} \cdot x_{i j}+\bar{c}_{i j} \cdot y_{i j}\right)
$$

As a result, we have the following formulation for forest stand aggregating harvest scheduling with multiple harvests:

$$
Z=\max _{W} \operatorname{tr}\left(\tilde{\boldsymbol{C}}^{\prime} \cdot \boldsymbol{W}\right)=\sum_{i=1}^{m} \sum_{j=1}^{n}\left(c_{i j} \cdot x_{i j}+\bar{c}_{i j} \cdot y_{i j}\right)
$$

subject to

$$
\left[\tilde{\boldsymbol{A}}+\operatorname{diag}\left(\tilde{\boldsymbol{A}} \cdot \mathbf{1}_{2 m n}\right)\right] \cdot \operatorname{vec}\left(\boldsymbol{W}^{\prime}\right) \leq \tilde{\boldsymbol{A}} \cdot \mathbf{1}_{2 m n}
$$

Adjacency constraints of Eq. [11] are necessary to generate aggregated management units exactly separated from other units as well as forest stands. Table 1 shows the proposed formulation for aggregating harvest scheduling problems compared to the one for non-aggregating spatially constrained problem allowing for multiple harvests from Yoshimoto and Konoshima (2015). The structure of this formulation is almost the same as that of Yoshimoto and Konoshima (2015).

\section{Demonstrative Example}

An example of a map from Sagawa Village of Kochi prefecture in Figure 1, which consists of 462 base units, demonstrates our formulation for forest stand aggregating harvest scheduling. We set the aggregation size to approximately 30 ha, which is most widely applied in Japan. Using a buffering approach from the center of each base unit, the rule allowed aggregation of adjacent base units into one hyper unit until the total area of the derived hyper unit was approximately equal to 30 ha. Each hyper unit was constructed by finding and aggregating all base units which are within a certain distance from the target unit. In this demonstration, we used $220 \mathrm{~m}$ as the target distance. Figure 2 
Table 1. Compact formulation of harvest scheduling for non-aggregation and aggregation.

Non-aggregation with multiple harvests (Yoshimoto and Konoshima, 2015)

Aggregation with multiple harvests

$$
Z=\max _{X} \operatorname{tr}\left(\boldsymbol{C}^{\prime} \cdot \boldsymbol{X}\right)=\sum_{i=1}^{m} \sum_{j=1}^{n} c_{i, j} \cdot x_{i, j}
$$

st.

$\left(\boldsymbol{O}^{\mathrm{U}} \otimes{\mathbf{1}_{n}^{\prime}}_{n}\right) \cdot \operatorname{vec}\left(\boldsymbol{X}^{\prime}\right) \leq \mathbf{1}_{m}$

$(1-\alpha) v_{0} \leq \operatorname{tr}\left(\boldsymbol{V}_{p}^{\prime} \cdot \boldsymbol{X}\right) \leq(1+\alpha) v_{0}, \quad p=1,2, \ldots, T$

$\left.\tilde{\boldsymbol{A}}+\operatorname{diag}\left(\tilde{\boldsymbol{A}} \cdot \mathbf{1}_{m n}\right)\right] \cdot \operatorname{vec}\left(\boldsymbol{X}^{\prime}\right) \leq\left(\boldsymbol{A}^{S} \cdot \mathbf{1}_{m}\right) \otimes \mathbf{1}_{n}$ $\tilde{\boldsymbol{A}}=\boldsymbol{A}^{S} \otimes \boldsymbol{A}^{V} \quad(m n \times m n)$ $Z=\max _{X} \operatorname{tr}\left(\tilde{\boldsymbol{C}}^{\prime} \cdot \boldsymbol{W}\right)=\sum_{i=1}^{m} \sum_{j=1}^{n}\left(c_{i, j} \cdot x_{i, j}+\bar{c}_{i, j} \cdot y_{i, j}\right)$

st.

$\left(\boldsymbol{O} \otimes{\mathbf{1}_{n}^{\prime}}_{n}\right) \cdot \operatorname{vec}\left(\boldsymbol{W}^{\prime}\right) \leq \mathbf{1}_{m}$

$(1-\alpha) v_{0} \leq \operatorname{tr}\left(\tilde{\boldsymbol{V}}_{p}^{\prime} \cdot \boldsymbol{W}\right) \leq(1+\alpha) v_{0}, \quad p=1,2, \ldots, T$

$\left.\tilde{\boldsymbol{A}}+\operatorname{diag}\left(\tilde{\boldsymbol{A}} \cdot \mathbf{1}_{2 m n}\right)\right] \cdot \operatorname{vec}\left(\boldsymbol{W}^{\prime}\right) \leq\left(\boldsymbol{A}^{S} \cdot 2_{m}\right) \otimes \mathbf{1}_{n}$

$\tilde{\boldsymbol{A}}=\boldsymbol{A}^{S} \otimes \boldsymbol{A}^{V} \quad(2 m n \times 2 m n)$

shows the histogram of the candidates for aggregation in terms of size by area. Candidates with less than 30ha were mainly due to the location of base units at the corner. The reward rate was set to $10 \%$. The objective was to maximize total harvested volume, subject to volume flow constraints with $10 \%$ fluctuation and extended land accounting as well as adjacency constraints. We used CPLEX (ILOG, 2003) to seek an optimal solution. We used the forest registration database ("Shinrin-bo") obtained from Kochi prefecture. Growth projection was conducted by estimating parameters of the Richards-Chapman growth function for each forest stand in the database.

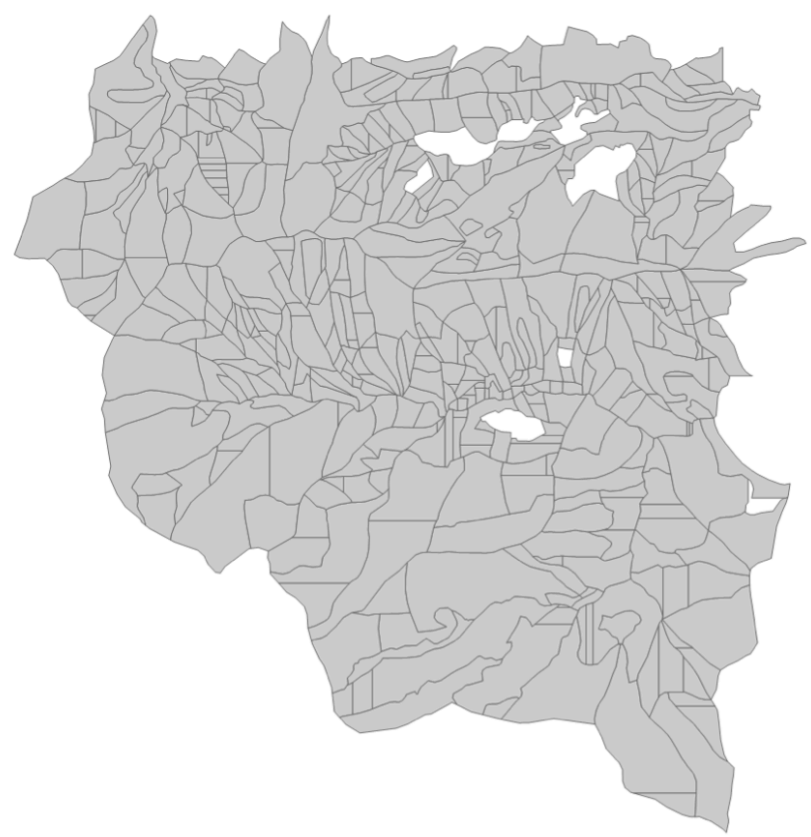

Figure 1. An example map with 462 base units.

$$
y=a\left(1-e^{-b \cdot t}\right)^{c}
$$

where $y$ is the stocking data $\left(\mathrm{m}^{3} / \mathrm{ha}\right), t$ is time (year), and $(a, b, c)$ is the set of parameters. Since in the database, there is only one point time growth stocking data, we applied Bayesian method by 


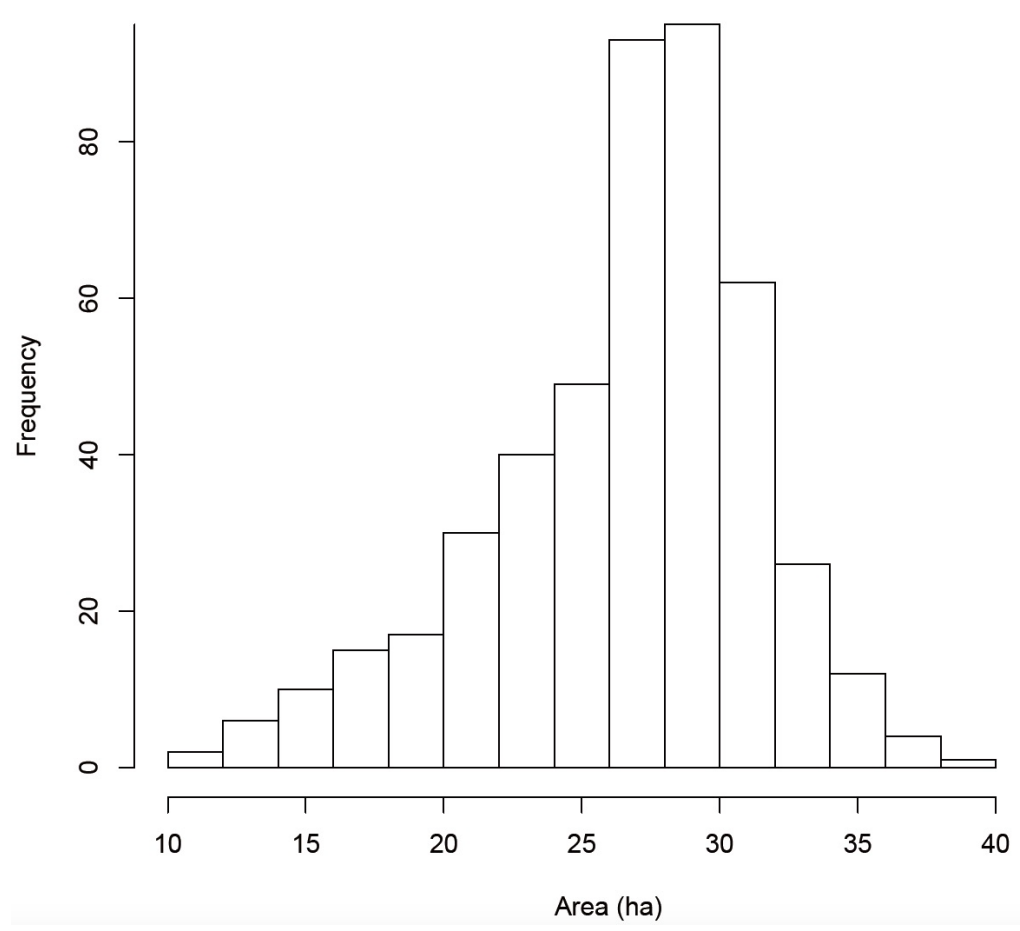

Figure 2. Histogram of candidates for aggregation based on $220 \mathrm{~m}$ distance buffering.

Ninomiya and Yoshimoto (2011) for estimation with the fitted results for all data as the prior of estimates. The prior estimates are updated through the iterative process for estimation until the data and prediction coincide with small tolerance of $10^{-4}$. Figure 3 shows the resultant growth projection estimated by Bayesian method applied here. We set the planning horizon to 10 periods, with 6 periods as the minimum rotation period. Possible 20 treatments were created for each forest stand as well as candidate management units.

Figure 4 shows the optimal solution that specifies the optimal spatial pattern of the hyper units, as well as base units not aggregated in any hyper unit. As for the number of hyper units, a minimum of one and a maximum of four were harvested during each planning period. In all, 15 hyper units were created in the optimal solution; among those, nine were harvested twice. Harvesting also took place in the base units during each period. A total of 73 base units were harvested and of those 21 were harvested twice. All base units were harvested at least once within the optimal solution over the planning horizon. To meet volume flow constraints in period 5, only one hyper unit was harvested along with 31 base units.

Our results show that by allowing harvest in either base units or hyper units, the optimal solution can be flexible to meet volume flow constraints over time. By considering multiple harvests over the planning horizon, forest resources can be utilized more efficiently. For instance, in our demonstrative example over 10 periods, 9 hyper units and 21 base units were harvested twice and all were harvested at least once by the end of the planning horizon. If we allowed only one harvest, the optimal spatial harvest pattern might look very different. Our experimental study demonstrates that the proposed formulation is a valid means of solving forest stand aggregation problem with multiple harvests.

\section{Concluding Remarks}

We proposed an alternative approach for optimal forest stand aggregation for implementing harvest scheduling that allows for multiple harvests using a compact formulated integer programming. The approach is alternative in a sense that a set of candidates for forest stand aggregation is predefined before the optimization process. The approach is to seek an optimal aggregated pattern among a set of candidates for forest management units over the planning horizon. We utilized the concept of a "hyper unit" or cluster as a possible aggregated management unit predefined with the use of ad- 


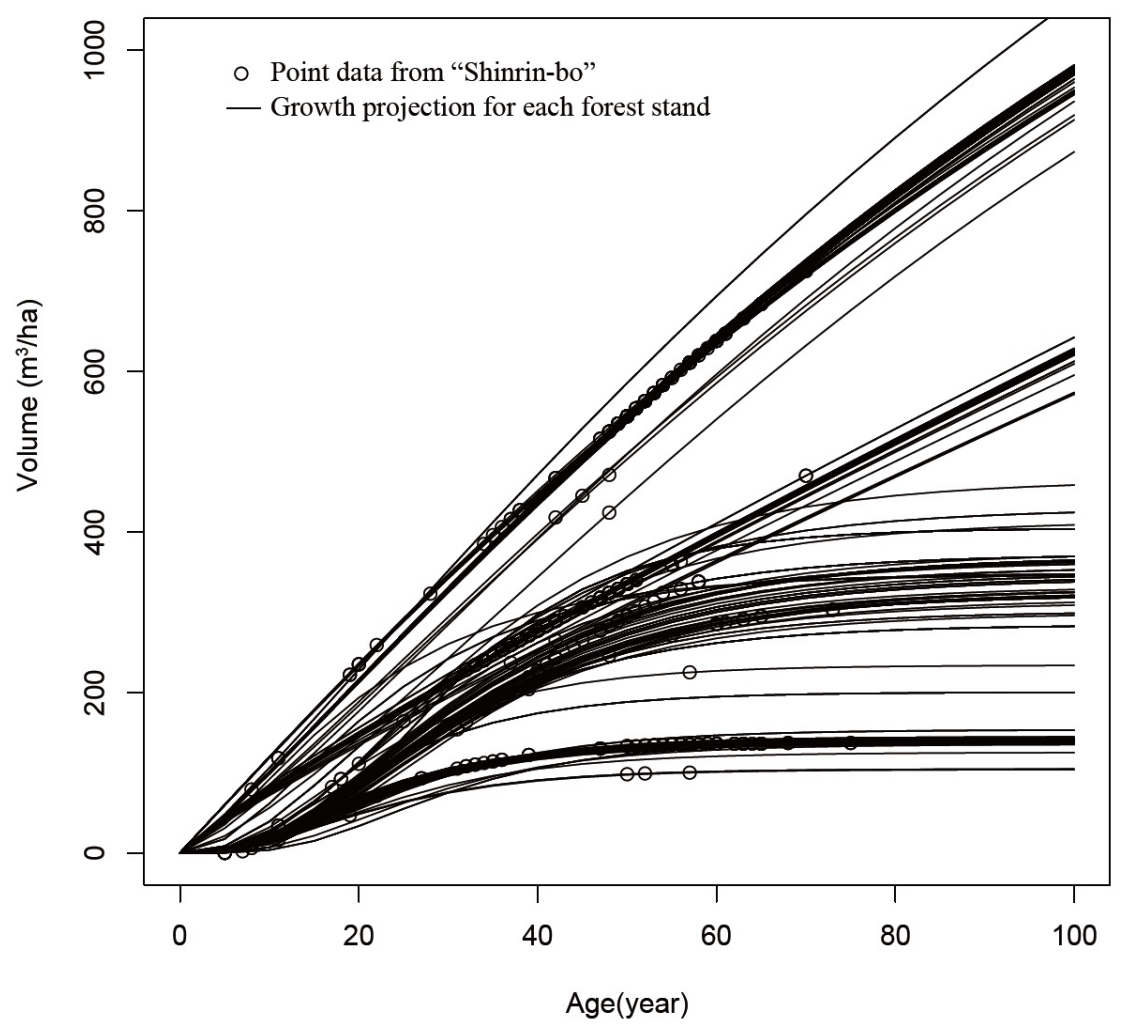

Figure 3. Growth projection for 462 base units based on Bayesian estimation.

jacency relationship among the set of forest stands, given a connection rule. Our proposed approach was based on the optimization framework of a traditional spatially constrained harvest scheduling problem which is used to choose the best set of treatments for the aggregated management units as well as the original un-aggregated forest stands allowing for multiple harvests. Adjacency constraints were also applied to create aggregated management units, which are separated from other units as well as un-aggregated forest stands, so that no adjacent stands are harvested in the same period.

We demonstrated our approach by using a map with 462 base units. With 30 ha set as aggregating size, our results showed that both hyper units and base units were harvested at least once over the planning period to completely utilize all base units. With this example we demonstrate that the proposed approach is valid for solving forest stand aggregation problem with multiple harvests, which surely helps local forest cooperatives to propose efficient and effective "Danchi-ka" location and scheduling.

It is worth noting that this approach can be applied not only for aggregating base units for harvest to improve the efficiency of management, but also for aggregating units to have a certain vegetation condition for habitat conservation. In our approach to create candidate hyper units, a pre-specified rule was applied. By modifying this rule, additional management objectives can be optimized. For example, suppose land managers would like to know the most efficient way to allocate habitat for a certain wildlife species that requires some kind of "connectivity". If there is information available on spatial conditions such as a vegetation type and suitable geographic conditions, as well as the minimum size required for "connected habitat", it is possible to develop a rule that would specify candidate aggregated units or hyper units to meet the requirements. The proposed approach could then be used to identify an optimal solution to allocate habitat among the candidate management units. 

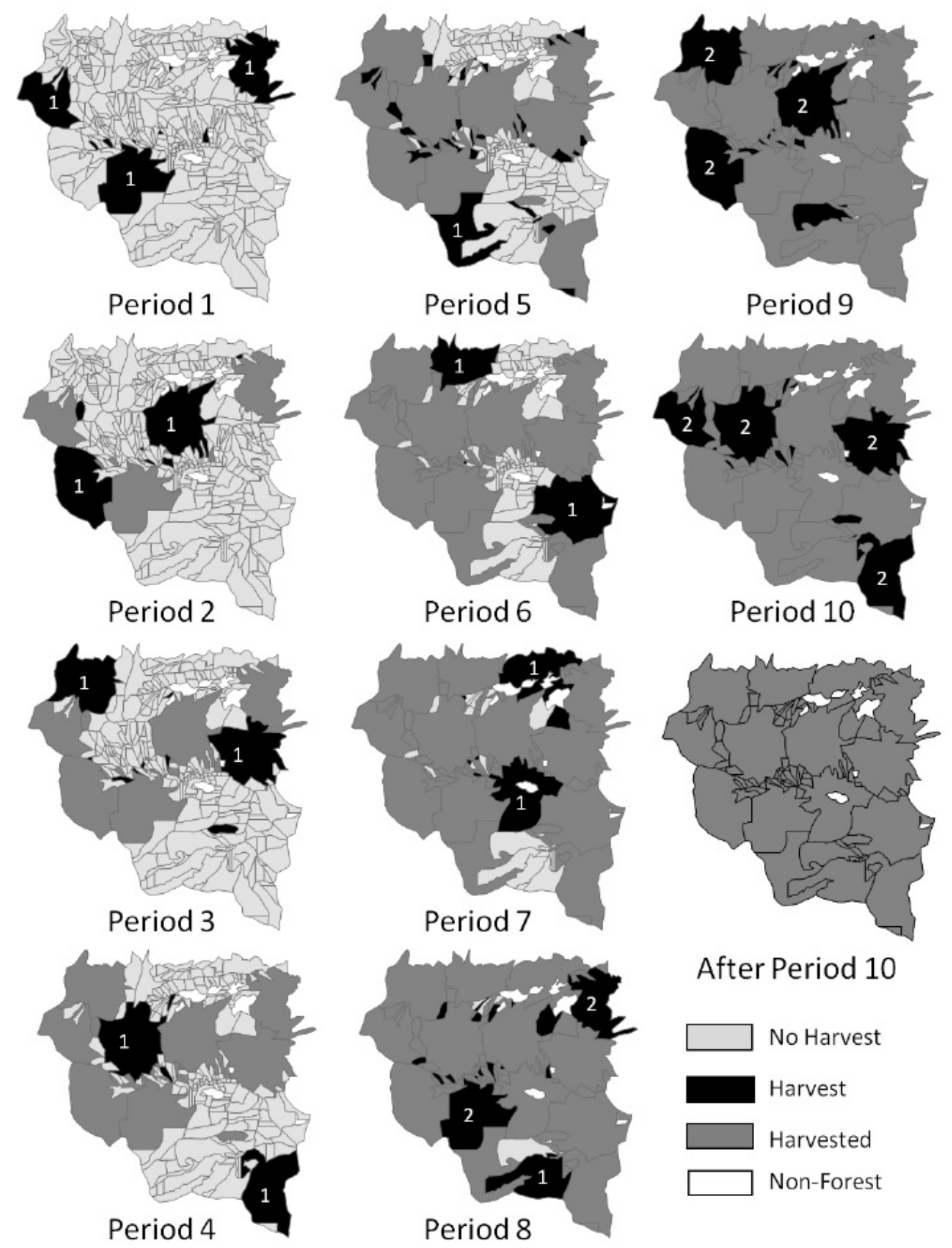

After Period 10
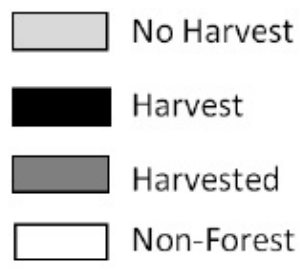

Figure 4. Optimal solution for hyper units and base units selected for harvesting. 


\section{Acknowledgement}

This research was funded by a Grant-in-Aid for Scientific Research from the Ministry of Education, Science, Sports, and Culture of Japan (Grant No.22252002).

\section{References}

Boston, K. and Bettinger, P. (2002) Combining tabu search and genetic algorithm heuristic techniques to solve spatial harvest scheduling problems, For. Sci. 48: 35-58.

Boston, K. and Bettinger, P. (2006) An economic and landscape evaluation of the green-up rules for California, Oregon, and Washington (USA), For. Pol. Econ. 8: 251-266.

British Columbia Ministry of Forests (1999) Forest Practices Code of British Columbia: Green-up Guidebook, Victoria, Province of British Columbia, pp. 37.

Goycoolea, M., Murray, A.T., Barahona, F., Epstein, R., and Weintraub, A. (2005) Harvest scheduling subject to maximum area restrictions: exploring exact approaches, Oper. Res. 53: 490-500.

Goycoolea, M., Murray, A., Vielma, J.P., and Weintraub, A. (2009) Evaluating approaches for solving the area restriction model in harvest scheduling, For. Sci. 55: 149-165.

ILOGS SA (2003) ILOGS CPLEX9.0 User's Manual, pp. 564.

Johnson, K.N. and Scheurman, H.L. (1977) Techniques for prescribing optimal timber harvest and investment under different objectives-discussion and synthesis, For. Sci. 23(1): a0001-z0001.

MAFF (2012) Annual report on forest and forestry in Japan for FY2011. Tokyo, Ministry of Agriculture, Forestry and Fisheries (in Japanese with English summary).

McDill, M.E., Rebain, S., and Braze, J. (2002) Harvest scheduling with area-based adjacency constraints, For. Sci. 48: 631-642.

Murray, A.T. (1999) Spatial restrictions in harvest scheduling, For. Sci. 45: 45-52.

Murray, A.T. and Weintraub, A. (2002) Scale and unit specification influences in harvest scheduling with maximum area restrictions, For. Sci. 48: 779-789.

Ninomiya, Y. and Yoshimoto, A. (2011) Bayesean method for predicting forest tree growth curve, FORMATH 10: 333-349 (in Japanese with English abstract).

Rebain, S. and McDill, M.E. (2003) A mixed-integer formulation of the minimum patch size problem, For. Sci. 49: 608-618.

Yoshimoto, A. (2001) Potential use of a spatially constrained harvest scheduling model for biodiversity concerns: exclusion periods to create heterogeneity in forest structure, J. For. Res. 6: $21-30$.

Yoshimoto, A. and Konoshima, M. (2015) Spatially constrained harvest scheduling for multiple harvests by exact formulation with common matrix algebra, J. For. Res. 21: 15-22. 